\title{
Proteomic Characterization Reveals a Molecular Portrait of Nasopharyngeal Carcinoma Differentiation
}

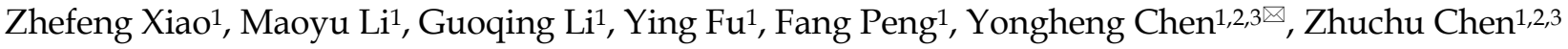 \\ 1. Key Laboratory of Cancer Proteomics of Chinese Ministry of Health, Xiangya Hospital, Central South University, Changsha, Hunan, P. R. China; \\ 2. State Key Laboratory of Medical Genetics, School of Life Sciences, Central South University, Changsha, Hunan, P.R. China; \\ 3. Collaborative Innovation Center for Cancer Medicine (CICCM), Guangzhou, Guangdong, P. R. China. \\ $\triangle$ Corresponding author: Yongheng Chen, Key Laboratory of Cancer Proteomics of Chinese Ministry of Health, Xiangya Hospital, 87 Xiangya Road, Changsha, \\ Hunan, 410008, P. R. China. Phone: 86-731-84327608. Email: yonghengchen@gmail.com. \\ (C) Ivyspring International Publisher. This is an open access article distributed under the terms of the Creative Commons Attribution (CC BY-NC) license \\ (https://creativecommons.org/licenses/by-nc/4.0/). See http://ivyspring.com/terms for full terms and conditions.
}

Received: 2016.08.31; Accepted: 2016.10.29; Published: 2017.02.11

\begin{abstract}
Nasopharyngeal carcinoma (NPC) is categorized into three different differentiated subtypes by World Health Organization (WHO). Based on an earlier comparative proteomic database of the three histological subtypes, the study was to deepen our understanding of molecular mechanisms associated with NPC differentiation through bio-information mining. Among the three subtypes were 194 differentially expressed proteins (DEPs) of 725 identified proteins. Two DEPs, heat shock protein family B (small) member 1 (HSPBI) and keratin 5 (KRT5), were validated in a series of NPC tissue samples by using immunohistochemistry. Quantified protein families including keratins, S100 proteins (S100s) and heat shock proteins exhibited characteristic expression alterations. Comparisons of predicted bio-function activation states among different subtypes, including formation of cellular protrusion, metastasis, cell death, and viral infections, were conducted. Canonical pathway analysis inferred that Rho GTPases related signaling pathways regulated the motility and invasion of dedifferentiated NPC. In conclusion, the study explored the proteomic characteristics of NPC differentiation, which could deepen our knowledge of NPC tumorigenesis and allow the development of novel targets of therapeutic and prognostic value in NPC.
\end{abstract}

Key words: nasopharyngeal carcinoma, formalin-fixed paraffin-embedded, proteomics, Ingenuity pathway analysis.

\section{Introduction}

Nasopharyngeal carcinoma (NPC) is an endemic epithelial neoplasm, with an annual incidence of 25-50 cases per 100,000 in southern China and Southeast Asia, though relatively rare in the Western world [1]. It differs from other head and neck cancers in its aetiology, epidemiology and potential therapeutic options [2]. Although NPC responds very well to radiotherapy, the outcome largely relates to the extent of the disease. Due to the deep location and vague symptoms, NPC is commonly diagnosed at a more advanced stage when possessing a higher metastatic potential, as compared to other head and neck carcinomas [3, 4]. For this reason, early diagnosis of NPC is critical for a better prognosis.

NPC is histologically categorized into three subtypes according to the World Health Organization
(WHO) classification, including keratinizing squamous cell carcinoma (type I), non-keratinizing squamous carcinoma (type II) and undifferentiated carcinoma (type III) [5]. From type I to III, NPC becomes less differentiated, more strongly associated with Epstein-Barr virus (EBV) infection and more sensitive to radiotherapy [6-8]. Correct histological classification plays a vital role in guiding treatment and improving prognosis of NPC.

In our previous study, an effective approach using two-dimensional liquid chromatography, and tandem mass spectrometry (2D LC-MS/MS) in conjunction with isobaric tags for relative and absolute quantitation (iTRAQ) labeling was performed to identify protein changes among the three histological subtypes of formalin-fixed 
paraffin-embedded (FFPE) NPC tissues [9]. The focus was on the evaluation of the effectiveness of the method and the similarity of the proteome inventories obtained from the FFPE and frozen tissue specimens. It was concluded that the obtained database from the diverse differentiation types of NPC tissues was reliable and effective.

In the present study, two more differentially expressed proteins (DEPs) were validated, and bioinformatics analysis of the whole DEPs from the data was performed by using Ingenuity Pathway Analysis (IPA) software. It not only extended our understanding of NPC differentiation, but also provided a list of biomarkers for histological classification, which might benefit the guidance of NPC treatment.

\section{Materials and Methods}

\section{Tissues}

This study was approved by institutional review board of the Cancer Hospital of Hunan Province. The set of FFPE tissue blocks of three WHO subtypes of $\mathrm{NPC}$, for immunohistochemical stain verification of specific proteins, were retrieved randomly from the Cancer Hospital of Hunan Province at the time of diagnosis before any therapy. It contained 11 cases of WHO type I ( 8 males and 3 females, age $41-72$ years, average $57 \pm 8$ years), 24 cases of WHO type II (19 males and 5 females, age 30-75years, average $51 \pm 13$ years), 30 cases of WHO type III (23 males and 7 females, age 26-74 years, average $51 \pm 12$ years), collected between 2000 and 2010 .

\section{Immunohistochemistry}

Four micrometer-thick tissue sections were deparaffinized in xylene, rehydrated in a graded ethanol series, and treated with antigen retrieval solution (10 mmol/L sodium citrate buffer; $\mathrm{pH}$ 6.0). The sections were incubated with rabbit anti-KRT5 monoclonal antibody (1:400, Epitomics, Burlingame, CA), or mouse anti-HSPB1 monoclonal antibody (1:200, Cell Signaling Technology, Danvers, MA) overnight at $4^{\circ} \mathrm{C}$. And then were incubated with 1:1000 dilution of biotinylated secondary antibody followed by avidin-biotin peroxidase complex (Dako, Carpinteria, CA) according to the manufacturer's instructions. Finally, tissue sections were incubated with 3', 3'-diaminobenzidine (Sigma Aldrich, St. Louis, MO) until a brown color developed, and counterstained with Harris' modified hematoxylin. In negative controls, primary antibodies were omitted.

HSPB1 and KRT5 staining were blindly scored by two investigators according to percentage of positive staining in the whole section for each case $(0$ $=$ no positive staining; $1=1 \%-30 \%$ positive; $2=$
$31 \%-60 \%$ positive; $3=61 \%-100 \%$ positive) and its intensity $(0=$ no staining, $1=$ mild staining, $2=$ moderate staining, 3 = intense staining), as described previously [10]. Finally a total score (ranging from 0-6) was obtained by adding the area score and the intensity score for each case. Care was taken not to interpret nasopharyngeal stroma cells as positive for tumor staining. Appropriate controls were also included. Kruskal-Wallis one way analysis of variance (ANOVA) test was used to calculate the $\mathrm{p}$ value for multiple comparisons, and it was considered statistically significant when $p<0.05$.

\section{Differentially Expressed Proteins Analysis}

The DEPs were selected based on the following criteria: protein identified with $\geq 2$ unique peptides with at least $95 \%$ confidence; proteins with iTRAQ ratios of above 1.5 or below 0.667 between any two groups of iTRAQ 115, 116, and 117. The list of all DEPs and their corresponding relative expression values were uploaded separately as three groups (iTRAQ 116/115, iTRAQ 117/115, iTRAQ 117/116) and analyzed on IPA software server (http://www.ingenuity.com, version 9.0, Ingenuity Systems, Redwood City, CA) for further analysis. The reference set and parameters for IPA on the DEPs list were as follows: (i) Reference set, Ingenuity Knowledge Base (Genes Only); (ii) Relationship to include, Direct and Indirect; (iii) Filter Summary, Consider molecules and/or relationships where $($ species $=$ All) AND $($ cell lines $=$ All tissues and cell lines in ingenuity database) AND (confidence =experimentally observed and high predicted).

The IPA downstream effects analysis predicts increases or decreases in downstream biological activities occurring in the samples, based upon the direction of change of the proteins. Predicted activation state is based on the strength of the $z$-score. $\mathrm{Z} \geq 2$ is "activated" and $\mathrm{z} \leq-2$ is "inhibited". Statistical significance is set at $p<0.05$. The main biological functions of the DEPs were collected from Uniprot protein knowledge database (http://www.uniprot. org) and PubMed (http://www.ncbi.nlm.nih.gov). Canonical pathway analysis identified the pathways ( $p$-value $\leq 0.01$ ), from the IPA library of canonical pathways.

\section{Results}

\section{Data presentation}

The database was obtained in our previous study [9]. The two MS/MS replicates resulted in 611 and 621 unique proteins respectively, by using the criteria of unused ProtScore $\geq 1.3$ with at least one peptide with $95 \%$ confidence. There were a total of 725 unique proteins after combined (Supporting Information 
Table S1). The proteins known to present in plasma including hemoglobins, serum albumins and apolipoprotein were neglected.

In all DEPs, 144 were found to be differentially expressed in type I and type II, 87 were differentially expressed between type II and type III, and 152 were differentially expressed between type I and type III (shown in bold in Supporting Information Table S1). The identified protein families including heat shock proteins, keratins, S100s, eukaryotic elongation factors (EEFs) and eukaryotic initiation factors (EIFs) showed characteristic expression variations, which were listed in Fig. 1. Most of the HSPs were up-regulated during NPC dedifferentiation, except for HSPB1 and HSPE1. All identified keratins showed a descendant alteration when NPC was losing differentiation. S100A7, S100A8 and S100A9 showed a significant decrease while S100A4 was found increased from type I to type III. EIF4A1, EIF4G1 and EEFs exhibited a progressively increasing expression.

\section{Validation of the Proteomic Results by Immunohistochemistry}

The expression alteration of three DEPs including cathepsin D (CTSD), keratin8 (KRT8) and
SFN (14-3-3 $\sigma)$ were consistent with their immunohistochemistry outcomes in primary NPC tissues with diverse differentiation degrees in our previous studies [10-12]. To cover more and further validate the findings, HSPB1 and KRT5 were included for immunohistochemistry analysis. The expression of KRT5 and HSPB1 were tested in 11 cases of WHO type I, 24 cases of WHO type II, and 30 cases of WHO type III. The expression levels of HSPB1 and KRT5 were significantly decreased in WHO type III as compared to type I. Representative immunohistochemistry staining of the two proteins in the three different histological subtypes were presented in Fig. 2. The stain scores for percentage and intensity of the proteins were presented in Table 1 in detail. Kruskal-Wallis one way ANOVA test confirmed that HSPB1 was expressed with statistically significant differences between any two histological subtypes. KRT5 expression level of type I was significantly different from that of type III. Though no obvious statistical significance was detected between type I and type II, the gradually reduced expression of KRT5 from type I to type III was clear.
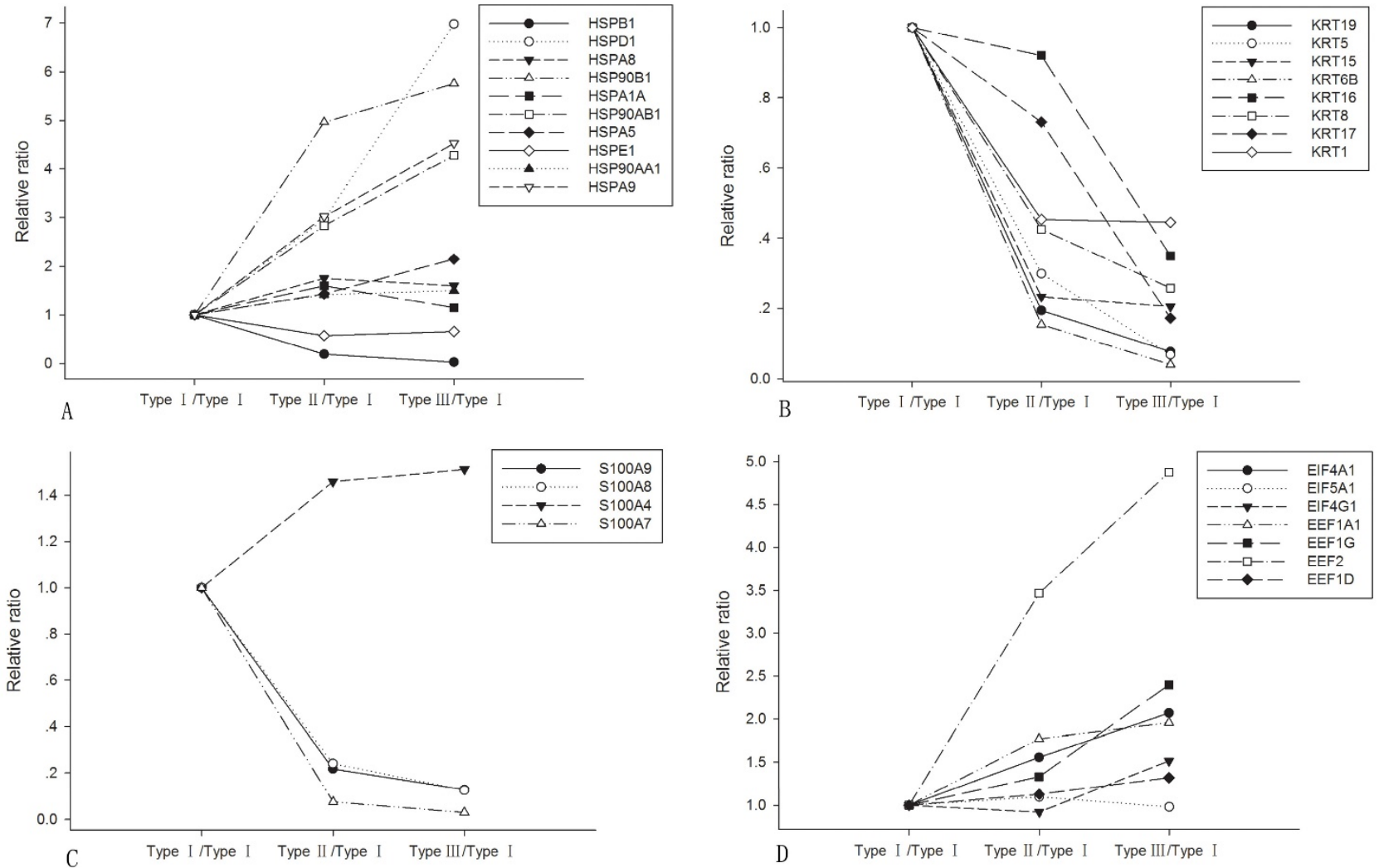

Figure 1. Expression alterations of the representative protein families among the three subtypes of NPC. A. heat shock proteins; B. keratins; C. S100As; D. EEFs and EIFs. 

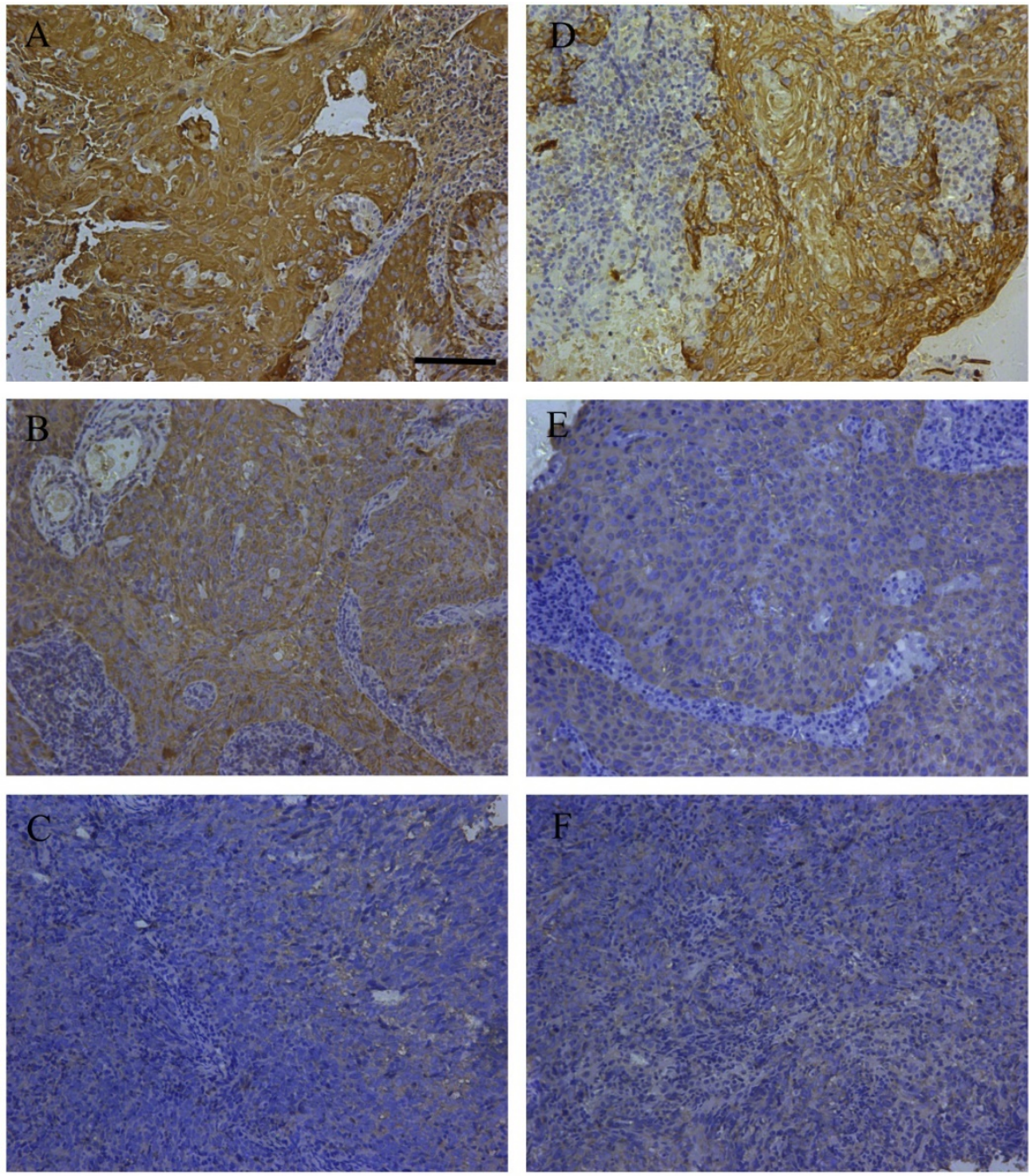

Figure 2. Representative immunohistochemistry results of HSPBI and KRT5 in clinical NPC samples. Immunohistochemistry of HSPBI in WHO type I (A), WHO type II (B), and WHO type III (C); immunohistochemistry of KRT5 in WHO type I (D), WHO type II (E), and WHO type III (F); bar size=100 $\mu \mathrm{m}$.

Table 1. Immunohistochemistry scores of HSPBI and KRT5 in different subtypes of NPC samples.

\begin{tabular}{|c|c|c|c|c|c|c|c|c|c|c|c|c|c|}
\hline \multirow{3}{*}{$\begin{array}{l}\text { Histological subtypes } \\
\text { (WHO) }\end{array}$} & \multirow{3}{*}{$\begin{array}{l}\text { Number } \\
\text { of cases }\end{array}$} & \multicolumn{6}{|c|}{ HSPB1 } & \multicolumn{6}{|c|}{ KRT5 } \\
\hline & & \multirow[t]{2}{*}{$0-2$} & \multirow[t]{2}{*}{$3-4$} & \multirow[t]{2}{*}{$5-6$} & \multicolumn{3}{|l|}{$p$} & \multirow[t]{2}{*}{$0-2$} & \multirow[t]{2}{*}{$3-4$} & \multirow[t]{2}{*}{$5-6$} & \multicolumn{3}{|l|}{$p$} \\
\hline & & & & & I vs II & II vs III & I vs III & & & & I vs II & II vs III & I vs III \\
\hline WHO I & 11 & 0 & 2 & 9 & 0.031 & 0.019 & 0.000 & 0 & 4 & 7 & $\mathrm{Na}$ & 0.009 & 0.003 \\
\hline WHO II & 24 & 2 & 16 & 6 & & & & 1 & 12 & 11 & & & \\
\hline WHO III & 30 & 13 & 15 & 2 & & & & 8 & 18 & 4 & & & \\
\hline
\end{tabular}

$p$ was calculated by Kruskal-Wallis one way ANOVA test.

\section{Bioinformatic analysis of the differentially expressed proteins}

Based on the change direction of the DEPs, the likely effect (increase or decrease) on cell biological processes were measured and predicted by IPA. The representative bio-functions related to NPC characteristics with statistical significance were shown in Table 2, including their predicted states and the supportive regulators that were quantified in our data. The activity of metastasis increased significantly in type III compared to type II, while cell death decreased apparently in type III compared to type I. Three bio-functions related to cellular assembly including cytoskeleton organization, microtubule dynamics and formation of cellular protrusions, all increased from type I to type III. Synthesis of adenosine triphosphate (ATP) was also elevated in type III. Viral infection was more aggravated from type II to type III. The increased activation of inhibitor of kappa B (I-kappa B) and nuclear factor kappa B (NF-kappa B) cascade was observed in type III when compared to type II. 
Table 2. Predicted states of the representative biological functions with statistical significance, followed by the involved and quantified proteins.

\begin{tabular}{|c|c|c|c|c|}
\hline Functions Annotation & p-Value & Predicted Activation State & Activation z-score & Molecules \\
\hline cell death of tumor cell lines & 9.19E-11 & Decreased (type III vs. type I) & -2.215 & $\begin{array}{l}\text { AGR2,ANXA2,APEX1,ATP5A1,CAPNS1,CBR1,CCT2,CNPY2,COX5A,CTSD, } \\
\text { DDX3X,ENO1,FUBP1,GAPDH,GNB2L1,GSTP1,H2AFX,HNRNPK,HSP90AB1 } \\
\text {,HSPA5,HSPA8,HSPA9,HSPB1,HSPD1,LGALS3,LMNB1,MIF,MSN,NCL,NP } \\
\text { M1,P4HB,PARP1,PKM,PRKDC,PSAP,PTPN6,PYCARD,RPS19,S100A4,S100A } \\
\text { 8,S100A9,SFN,SRSF1,TAGLN2,TYMP,VCP,XRCC5 }\end{array}$ \\
\hline metastasis & 2.65E-07 & Increased (type III vs. type II) & 2.359 & $\begin{array}{l}\text { AGR2,ANXA1,CAPG,CBR1,COL6A3,FLNA,FUS,HSP90AB1,KRT8,LGALS1,L } \\
\text { GALS3,LTF,MIF,MX1,PRDX2,SERPINA1,TUBA4A,TUBB4B }\end{array}$ \\
\hline organization of cytoskeleton & 2.30E-05 & Increased (type III vs. type I) & 2.784 & $\begin{array}{l}\text { ACTR3,ANXA1,CALML3,CANX,CAP1,CAPG,CAPNS1,CFL1,CTNND1,EEF } \\
\text { 1A1,GAPDH,HSP90AA1,HSPB1,IQGAP1,KPNB1,KRT16,KRT17,LCP1,LTF,M } \\
\text { SN,MYH9,NUMA1,PDIA3,PFN1,PKM,RAP1B,S100A4,S100A9,STIP1,TLN1,V } \\
\text { IM }\end{array}$ \\
\hline microtubule dynamics & $1.41 \mathrm{E}-04$ & Increased (type III vs. type I) & 2.724 & $\begin{array}{l}\text { ACTR3,CALML3,CANX,CAPG,CAPNS1,CFL1,CTNND1,EEF1A1,GAPDH,H } \\
\text { SP90AA1,HSPB1,IQGAP1,KPNB1,LCP1,LTF,MSN,MYH9,NUMA1,PDIA3,PF } \\
\text { N1,PKM,RAP1B,S100A4,STIP1,TLN1,VIM }\end{array}$ \\
\hline $\begin{array}{l}\text { formation of cellular } \\
\text { protrusions }\end{array}$ & 5.12E-03 & Increased (type III vs. type I) & 2.939 & $\begin{array}{l}\text { ACTR3,CAPG,CAPNS1,CFL1,CTNND1,EEF1A1,HSP90AA1,HSPB1,IQGA } \\
\text { P1,LCP1,MSN,PDIA3,PFN1,RAP1B,S100A4,STIP1,VIM }\end{array}$ \\
\hline synthesis of ATP & 7.85E-04 & Increased (type III vs. type I) & 2 & ATP5B,FBP1,HSPD1,PARP1,PKM \\
\hline viral infection & 3.87E-10 & Increased (type III vs. type II) & 2.025 & $\begin{array}{l}\text { ANXA1,ANXA6,ATP5B,BGN,FLNA,GAA,GANAB,GAPDH,GBP1,GSTO1,HI } \\
\text { ST1H2BD,HSP90AB1,HSPA5,HSPA9,HSPD1,LGALS1,LGALS3,LMNA,LTF, } \\
\text { MIF,MX1,NCL,PDIA3,PRDX2,PSMC3,PYCARD,RPL12,RPS13,RPS5,S100A8,S } \\
\text { 100A9,SAMHD1,SERPINA1,SFN,STAT1,SUB1,TAGLN2,TUBA4A,TUBB4B }\end{array}$ \\
\hline $\begin{array}{l}\text { I-kappa B kinase/NF-kappa } \\
\text { B cascade }\end{array}$ & 2.87E-03 & Increased (type III vs. type II) & 2 & FLNA,HSPB1,LGALS1,PYCARD,STAT1 \\
\hline
\end{tabular}

To further clarify how the downstream bio-function states were measured and predicted based on the variation directions of the DEPs, an example highlighted in Table 2 was explicated in Fig. 3. Formation of cellular protrusions was predicted to be activated in type III NPC when compared to type I, which was contributed by the increased expression of actin-related proteins 3 (ACTR3), vimentin (VIM), stress induced phosphoprotein 1 (STIP1), member of RAS oncogene family (RAP1B), profiling 1 (PFN1), lymphocyte cytosolic protein 1 (LCP1), IQ motif containing GTPase activating protein 1 (IQGAP1), HSP90AA1, EEF1A1 and catenin delta 1 (CTNND1), and the decreased expression of calpain small subunit 1 (CAPNS1). The prediction of the bio-function activation was statistically significant (z-score $=2.939$, $p$ value $=0.00512$ ). Of note, of 11 effective regulators, 8 showed approximately linear expression alterations. It suggested an agreeable elongated morphology alteration from NPC type I to type III, which was corresponding to what was observed under a microscope.

Since the type III/type I group contains the most DEPs, it was focused on for canonical pathway analysis. The pathways ( $p$-value $\leq 0.01$ ) that shared three or more molecules were enriched and supposed to have cross connections and function overlapping. There appeared a large cluster of 6 related pathways including actin cytoskeleton signaling, epithelial adherens junction signaling, RhoA signaling, regulation of actin-based motility by Rho, Rho GDP dissociation inhibitor (Rho GDI) signaling, and signaling by Rho Family GTPases. All of the DEPs involved in the cluster were listed in Table 3.
Table 3. A list of all the quantified proteins involved in the cluster of six pathways. The list contains the quantification information from combination of the two MS/MS replicates of the ITRAQ experiment.

\begin{tabular}{|c|c|c|c|c|}
\hline \multirow{2}{*}{$\begin{array}{l}\text { Protein } \\
\text { Name }\end{array}$} & \multirow{2}{*}{$\begin{array}{l}\text { Unused } \\
\text { ProtScore }\end{array}$} & \multirow{2}{*}{$\begin{array}{l}\text { Peptides } \\
(95 \%)\end{array}$} & \multicolumn{2}{|l|}{ iTRAQ values } \\
\hline & & & Type II vs. Type I & Type III vs. Type I \\
\hline ACTR3 & 8.02 & 5 & 2.58 & 1.82 \\
\hline CFL1 & 8.01 & 5 & 1.53 & 1.57 \\
\hline CTNND1 & 4.13 & 2 & 3.56 & 3.25 \\
\hline GDI2 & 10.01 & 5 & 5.65 & 5.3 \\
\hline GNB2L1 & 4.89 & 3 & 1.56 & 1.63 \\
\hline IQGAP1 & 6.47 & 4 & 4.53 & 4.61 \\
\hline MRCL3 & 8.21 & 6 & 0.64 & 0.57 \\
\hline MSN & 12.04 & 5 & 3.1 & 1.92 \\
\hline MYH9 & 26.12 & 15 & 9.12 & 8.17 \\
\hline MYL6 & 4.01 & 5 & 0.61 & 0.76 \\
\hline PFN1 & 10.03 & 10 & 2.38 & 1.72 \\
\hline RAP1B & 4 & 3 & 2.01 & 1.87 \\
\hline TLN1 & 14.55 & 9 & 5.97 & 4.13 \\
\hline TUBA4A & 3.7 & 22 & 0.9 & 0.6 \\
\hline
\end{tabular}

Interestingly, almost half of them were exactly the regulators involved in the predicted activated formation of cellular protrusions between type I and type III that were listed in Fig.3. Though no direct Rho protein members were identified, deregulation of the downstream effectors suggested that Rho GTPase related signaling played a critical role in NPC cellular morphology transition and migration during dedifferentiation.

\section{Discussion}

Nasopharyngeal carcinoma is categorized into three histological subtypes, WHO type I, II and III. Proteomic analysis on NPC differentiation used to be 
hindered by the deficiency of NPC type I specimens in frozen form. The comparative proteomic data of the three subtypes was obtained in our earlier study [9]. In the present report, the data was released and bio-information mining was tried to deepen our understanding of NPC differentiation mechanisms.

By IPA downstream effects analysis, declined "cell death" and advanced "metastasis" in the undifferentiated subtype suggested that type III became more and more aggressive and malignant. Invading cells could be accompanied by morphological changes and it was also upheld by our quantitative data that pointed to more activated microtubule dynamics and formation of cellular protrusions in type III.

The downstream effectors of the clustered pathways identified and quantified in our study might provide some hints on the cell migration mechanisms during NPC dedifferentiation. IQGAP1 was significantly over-expressed during NPC dedifferentiation, which was found to destabilize E-cadherin-mediated cell-cell adhesion in pancreatic carcinoma cells, and lead to polarised cell morphology and directional cell migration [13, 14]. Interestingly, Calmodulin was detected decreased in our dataset, in accordance with a report that selective inhibition of Calmodulin function at the plasma membrane enhanced cell migration mediated by IQGAP1 [15]. Cofilin 1 (CFL1), One of the actin-modulating protein downstream targets of Rho GTPases, was up-regulated greatly from type I to type
III, whose activity was required for tumor cell motility and invasion [16]. Over-expression of CTNND1 (p120ctn), which was quantified in our dataset, was reported inducing a dendritic morphology in fibroblasts and increasing the activity of endogenous cell division cycle 42 (CDC42) and ras-related C3 botulinum toxin substrate 1 (RAC1), thereby promoting cell migration [17]. Talin 1 (TLN1), which showed a raised expression in poor differentiated NPC, mediated linkage of integrins to the actin cytoskeleton in focal adhesion complexes essential for cell adhesion and motility, and suppressed expression of E-cadherin, independent of integrin [18]. The expression of receptor for activated $C$ kinase 1 (GNB2L1 or RACK1) was found gradually elevated during NPC dedifferentiation. It was reported that up-regulation of RACK1 in non-small-cell lung cancer samples was inversely correlated with differentiation of the tumors and positively correlated with tumor metastasis [19]. RAP1B, over-expressed during NPC deteriorated differentiation, was known to play a role in the progression of angiogenesis and migration [20]. Apparently increased expression of VIM, along with the consistently decreased keratins from type I to type III suggested that the squamous carcinoma cells were losing their keratinizing epithelial characteristics, getting mesenchymal-like phenotypes, and exhibiting a highly invasive, epithelial-mesenchymal transition (EMT)-like histological pattern.

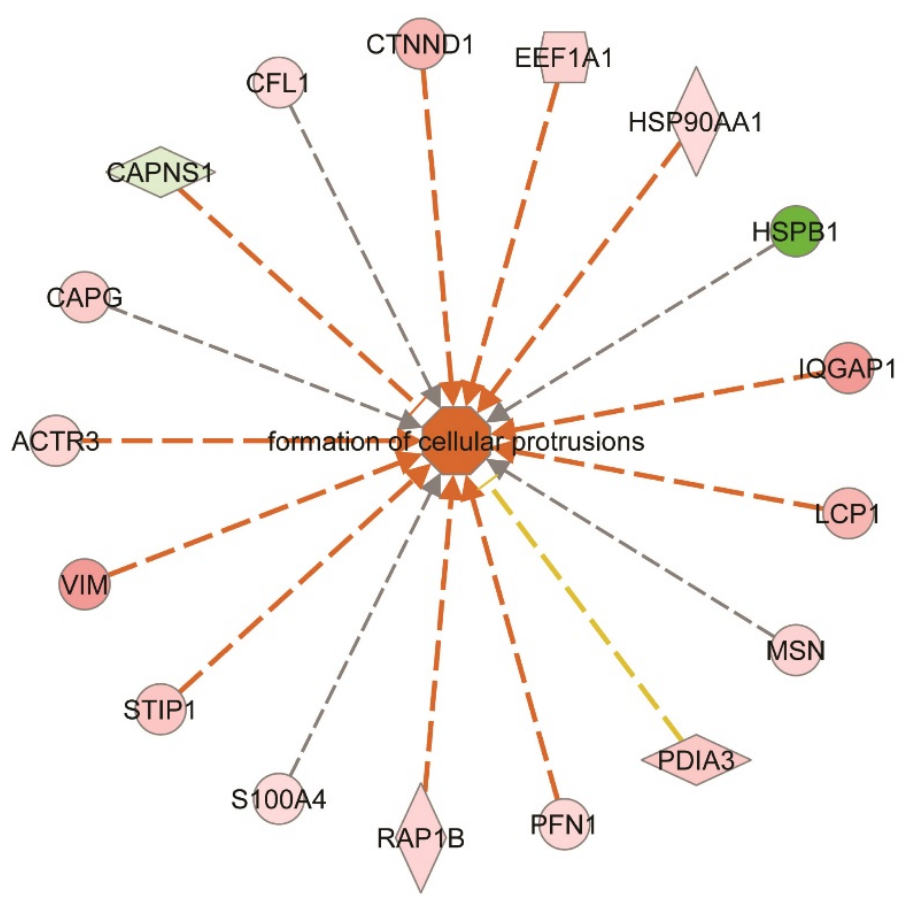

Prediction Legend more extreme less

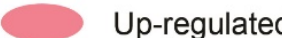

Down-regulated

Predicted activation

Predicted Relationships

Leads to activation

Effect not predicted

Findings inconsistent

with state of down-

stream molecule

Figure 3. The predicted activation of formation of cellular protrusions between type I and III NPCs is explained by the contribution of the quantified proteins. 
Four S100 proteins were detected in our study. S100A4 was up-regulated, while S100A7-9 kept decreasing during NPC dedifferentiation. S100A4 was a known mediator of EMT, leading to metastasis [21]. The expression of S100A7 was found associated with well differentiated head and neck squamous cell carcinoma [22]. S100A8 and S100A9 shared a strikingly similar decreasing expression pattern in our dataset, which was supported by the finding that they could form a heterodimeric complex and were usually co-expressed [23]. They were detected co-locating on chromosome 1q21 and forming a gene complex with the epidermal differentiation markers such as profilaggrin and involucrin [24]. Their attenuated expression was accompanying NPC declining differentiation.

Numerous HSP family members were quantified in our study, with molecule sizes varying from $10 \mathrm{kDa}$ to $90 \mathrm{kDa}$. Most of the HSPs including HSPD1, HSP90B1, and HSPA9 were increased from type I to type III of NPC, while HSPB1 was apparently down-regulated. Immunohistochemistry staining was further carried out and the down-regulation of HSPB1 from type I to type III was confirmed. HSPs were usually induced by stress and commonly elevated in cancer cells [25], while HSPB1 as an exception in our study might suggest its additional function. It was reported to be associated with higher differentiation in squamous carcinomas of the uterine cervix and oral epithelium [26]. Expression of HSPB1 also correlated with tumor differentiation in esophageal squamous cell carcinoma [27]. HSPB1 has been reported as a radioresistance-related protein in the NPC cell lines, inhibition of which enhanced radiosensitivity [28]. Accordingly, the reduced expression of HSPB1 in the poorly differentiated might associate with the clinical relevance of the NPC histological types that differentiation in fact conferred a worse response to radiotherapy in endemic area [8].

Increased EIF4A1, EIF4G1, and EEF family members including EEF2, EEF1G and EEF1A1 suggested the link between deregulation of protein synthesis and malignant behaviors. EEF2 was reported over-expressed in the majority of several types of cancers including lung, esophageal, breast and prostate cancers, and head and neck squamous cell carcinoma, which played an oncogenic role in cancer cell growth [29]. Over-expression of EIF4G1 was found correlated with NPC progression [30]. EIF4A1 was an early marker of distant metastases of non-small cell lung cancer [31].

In conclusion, with the help of IPA, individual DEPs were enriched and their expression variation was calculated to reflect the change of bio-function activation states and the canonical pathways related to NPC differentiation, thus deepening our knowledge of NPC tumorigenesis. Of note, besides expression levels, proteins were also subject to subcellular localization and posttranslational modification which need further study. More evaluation and investigation are required for the development of novel tumor therapeutic strategies targeting the representative protein families and special signaling pathways described in our study to treat NPC.

\section{Supplementary Material}

Table S1. http:/ / www.jcancer.org/v08p0570s1.xls

\section{Acknowledgments}

The authors thank the Department of Chemistry and the Institute of Biomedical Science, Fudan University, Peoples Republic of China, and CloudScientific Technology Co., Ltd, Shanghai, Peoples Republic of China, for technical support, and thank Dr. Meng Xia for discussion and help in manuscript preparation.

This work was supported by grants from the National Nature Science

Foundation of China (Nos 81202129, 81372904 and 81272971) and supported by Major State Basic Research Development Program of China (973 Program: No. 2014CBA02000-4).

\section{Competing Interests}

The authors have declared that no competing interest exists.

\section{References}

1. Kamran SC, Riaz N, Lee N. Nasopharyngeal carcinoma. Surg Oncol Clin N Am. 2015; 24: 547-61.

2. Razak AR, Siu LL, Liu FF, et al. Nasopharyngeal carcinoma: the next challenges. Eur J Cancer. 2010; 46: 1967-78.

3. Lo KW, To KF, Huang DP. Focus on nasopharyngeal carcinoma. Cancer Cell. 2004; 5: 423-8.

4. Yeh SA, Tang Y, Lui CC, et al. Treatment outcomes and late complications of 849 patients with nasopharyngeal carcinoma treated with radiotherapy alone. Int J Radiat Oncol Biol Phys. 2005; 62: 672-9.

5. Shanmugaratnam K. Histological typing of nasopharyngeal carcinoma. IARC Sci Publ. 1978: 3-12.

6. Chen CL, Wen WN, Chen JY, et al. Detection of Epstein-Barr virus genome in nasopharyngeal carcinoma by in situ DNA hybridization. Intervirology. 1993; 36: 91-8

7. Cheng SH, Tsai SY, Horng CF, et al. A prognostic scoring system for locoregional control in nasopharyngeal carcinoma following conformal radiotherapy. Int J Radiat Oncol Biol Phys. 2006; 66: 992-1003.

8. Cheung F, Chan O, Ng WT, et al. The prognostic value of histological typing in nasopharyngeal carcinoma. Oral Oncol. 2012; 48: 429-33.

9. Xiao Z, Li G, Chen Y, et al. Quantitative proteomic analysis of formalin-fixed and paraffin-embedded nasopharyngeal carcinoma using iTRAQ labeling, two-dimensional liquid chromatography, and tandem mass spectrometry. J Histochem Cytochem. 2010; 58: 517-27.

10. Cheng AL, Huang WG, Chen ZC, et al. Identification of novel nasopharyngeal carcinoma biomarkers by laser capture microdissection and proteomic analysis. Clin Cancer Res. 2008; 14: 435-45.

11. Cheng $\mathrm{AL}$, Huang WG, Chen $\mathrm{ZC}$, et al. Identificating cathepsin $\mathrm{D}$ as a biomarker for differentiation and prognosis of nasopharyngeal carcinoma by laser capture microdissection and proteomic analysis. J Proteome Res. 2008; 7: 2415-26. 
12. Cheng AL, Huang WG, Zhang PF et al. Comparative proteomics analysis of LCM purified nasopharyngeal carcinoma and normal nasopharyngeal epithelial tissue. Prog Biochem Biophys. 2008; 35:521-530

13. Hage B, Meinel K, Baum I, et al. Rac1 activation inhibits E-cadherin-mediated adherens junctions via binding to IQGAP1 in pancreatic carcinoma cells. Cell Commun Signal. 2009; 7: 23.

14. Mataraza JM, Briggs MW, Li Z, et al. IQGAP1 promotes cell motility and invasion. J Biol Chem. 2003; 278: 41237-45.

15. Mataraza JM, Li Z, Jeong HW, et al. Multiple proteins mediate IQGAP1-stimulated cell migration. Cell Signal. 2007; 19: 1857-65.

16. Wang W, Mouneimne G, Sidani M, et al. The activity status of cofilin is directly related to invasion, intravasation, and metastasis of mammary tumors. J Cell Biol. 2006; 173: 395-404.

17. Noren NK, Liu BP, Burridge $\mathrm{K}$, et al. p120 catenin regulates the actin cytoskeleton via Rho family GTPases. J Cell Biol. 2000; 150: 567-80.

18. Becam IE, Tanentzapf G, Lepesant JA, et al. Integrin-independent repression of cadherin transcription by talin during axis formation in Drosophila. Nat Cell Biol. 2005; 7: 510-6.

19. Shi S, Deng YZ, Zhao JS, et al. RACK1 promotes non-small-cell lung cancer tumorigenicity through activating sonic hedgehog signaling pathway. J Biol Chem. 2012; 287: 7845-58.

20. Chrzanowska-Wodnicka M, Kraus AE, Gale D, et al. Defective angiogenesis, endothelial migration, proliferation, and MAPK signaling in Rap1b-deficient mice. Blood. 2008; 111: 2647-56.

21. Rasanen K, Sriswasdi S, Valiga A, et al. Comparative secretome analysis of epithelial and mesenchymal subpopulations of head and neck squamous cell carcinoma identifies S100A4 as a potential therapeutic target. Mol Cell Proteomics. 2013; 12: 3778-92.

22. Tripathi SC, Matta A, Kaur J, et al. Nuclear S100A7 is associated with poor prognosis in head and neck cancer. PLoS One. 2010; 5: e11939.

23. Donato R. S100: a multigenic family of calcium-modulated proteins of the EF-hand type with intracellular and extracellular functional roles. Int J Biochem Cell Biol. 2001; 33: 637-68.

24. Mischke D, Korge BP, Marenholz I, et al. Genes encoding structural proteins of epidermal cornification and S100 calcium-binding proteins form a gene complex ("epidermal differentiation complex") on human chromosome 1q21. J Invest Dermatol. 1996; 106: 989-92.

25. Sarto C, Binz PA, Mocarelli P. Heat shock proteins in human cancer. Electrophoresis. 2000; 21: 1218-26.

26. Ciocca DR, Calderwood SK. Heat shock proteins in cancer: diagnostic, prognostic, predictive, and treatment implications. Cell Stress Chaperones. 2005; 10: 86-103.

27. Xue L, Yang L, Jin ZA, et al. Increased expression of HSP27 inhibits invasion and metastasis in human esophageal squamous cell carcinoma. Tumour Biol. 2014; 35: 6999-7007.

28. Zhang B, Qu JQ, Xiao L, et al. Identification of heat shock protein 27 as a radioresistance-related protein in nasopharyngeal carcinoma cells. J Cancer Res Clin Oncol. 2012; 138: 2117-25.

29. Oji Y, Tatsumi N, Fukuda M, et al. The translation elongation factor eEF2 is a novel tumorassociated antigen overexpressed in various types of cancers. Int $\mathrm{J}$ Oncol. 2014; 44: 1461-9.

30. Tu L, Liu Z, He X, et al. Over-expression of eukaryotic translation initiation factor 4 gamma 1 correlates with tumor progression and poor prognosis in nasopharyngeal carcinoma. Mol Cancer. 2010; 9: 78.

31. Ji P, Diederichs $\mathrm{S}$, Wang $\mathrm{W}$, et al. MALAT-1, a novel noncoding RNA, and thymosin beta4 predict metastasis and survival in early-stage non-small cell lung cancer. Oncogene. 2003; 22: 8031-41. 